\title{
Critical Success Factors for Cloud Migration in Higher Education Institutions: A Conceptual Framework
}

\author{
Abdulrahman Alharthi ${ }^{\mathrm{a}, \mathrm{b}}$, Madini O. Alassafi ${ }^{\mathrm{a}}$, Abdullah I. Alzahrani ${ }^{\mathrm{a}}$, Robert J Walters ${ }^{\mathrm{a}}$, Gary B. Wills ${ }^{\mathrm{a}}$ \\ ${ }^{a}$ University of Southampton, Southampton, UK \\ ${ }^{b}$ University of Jeddah, Jeddah, Saudi Arabia
}

\begin{abstract}
In the last few years, the cloud-computing paradigm experienced a considerable growth, making it the de-facto technology fueling almost all major online services. Nowadays, Universities have become more reliant on Information and Communication Technology (ICT) to provide educational services for their stakeholders. While most of the Universities in Europe and North America has harnessed the capabilities of cloud services to provide large scale flexibly electronic educational services. In Saudi Arabia, Most of the Universities are start-ups with limited IT Staff and amateur ICT infrastructure. Therefore, migrating their ICT services to cloud computing could deliver substantial benefits in the form of rich education content, increased efficiency, and agility that can be used to transform higher education in Saudi universities. However, migrating the Universities resources to third party platforms as cloud computing has pitfalls need to be considered carefully prior to the transition. Therefore, to successfully migrate to the cloud in the Saudi universities context, it is essential to identify the enabler factors that contribute toward a successful migration to the cloud environment in the higher education settings. Therefore, this research paper aims to propose a framework to investigate technological and organizational success factors that enable the migration process of the Saudi Universities' ICT to the cloud environment.
\end{abstract}

\section{Introduction}

Cloud computing is a model for enabling convenient, on-demand network access to a shared pool of configurable computing resources (e.g., networks, servers, storage, applications and services) that can be rapidly provisioned and released with minimal management effort or service provider interaction. This IT as utility paradigm was a dream of McCarthy back in 1960s [1]. In recent Years, advances in the Internet bandwidth have accelerated the use of cloud-based services by higher education institutions to supply their stakeholders with agile, scalable and on demand online educational services. Cloud computing offers several benefits for the educational institutions as follows: (1) Hardware cost reduction as the cloud is on-demand and "pay-peruse" fashion, it is more beneficial for universities than the investment of an expensive and limited infrastructure, as the utilization of such an infrastructure might be very low at certain times during the academic year. (2) Scalable storage and sharing as learning outcomes and resources can be stored in the cloud, which provides almost unlimited store and computation capacities. Documents can be commonly edited and shared in the cloud, some of the Public sharing services are provided by Google docs, live SkyDrive, and Office Live [2], (3) Universal access to the computational resources, as the learners can study and access the resources anywhere, anytime and from any device without setup efforts.

The demand for higher education in Saudi Arabia is growing steadily. However, the quality of higher education is a growing concern for Ministry of Higher Education. The rapid growth of the young population in Saudi Arabia requires the universities to improve their ICT resources as soon as they can to provide the students and the academics with the ICT resources they need to enhance their teaching and learning experience. This is further bolstered by the fact that cloud-computing resources can be scaled dynamically thereby provide non-restricted computing power for students as well as the academic. There is a need for agile, blended and flexible way to enhance the teaching and learning process to promote good quality research and job opportunities [3]. In Saudi higher education, several studies revealed a positive attitude for students, faculty, and university administrations toward the use of internet-based cloud services in Saudi Arabia [4]. However, despite the benefits cloud services can add to the universities ICT and the positive attitude towards the cloud usage in the Saudi Universities, the migration rate of cloud computing is still in the early stages. This is due to the fact that the migration process to the cloud may not be an easy task, As There is a wide range of challenges quoted in the literature including infrastructural, cultural and organizational factors that impede a successful IT project deployment in Saudi organizations [5].

Therefore, this proposed framework is aimed to investigate the factors that will affect the success of the migration project to the cloud. 


\section{Background of Cloud Computing}

Cloud Computing has evolved from technologies such as virtualisation, grid computing, distributed computing; web 2.0 technologies, Service Oriented Architecture and utility computing. There are many definitions for Cloud Computing in the literature. The three most cited definitions are presented below.

Buyya et al. defined Cloud Computing as "A type of parallel and distributed system consisting of a collection of interconnected and virtualized computers that are dynamically provisioned and presented as one or more unified computing resources based on service-level agreements established through negotiation between the service provider and consumers" [6].

Vaquero et. al. define Cloud Computing as: "a broad array of web-based services aimed at allowing users to obtain a wide range of functional capabilities on a 'pay-as-you-go' basis that previously required tremendous hardware/software investments and professional skills to acquire" [5].

National Institute of Standards and Technology (NIST) defines Cloud Computing as "a model for enabling convenient, on-demand network access to a shared pool of configurable computing resources (e.g., networks, servers, storage, applications and services) that can be rapidly provisioned and released with minimal management effort or service provider interaction".

\subsection{Cloud Characteristics}

According to the NIST definition, Cloud Computing has five characteristics as follows:

- On-demand-self-service: In Cloud Computing, users can automatically utilise computing resources such as servers, software, and storage as desired without any human interaction with a cloud service provider [7].

- $\quad$ Resource pooling: The cloud provider's pool of computing resources is grouped together to serve multiple tenants/clients in such a way that different physical and virtual resources are automatically allocated and relocated according to the user's demands [7].

- Broad network access: Resources in Cloud Computing are reachable over the Internet by standard techniques and used by heterogeneous thin or thick consumers' platforms [7].

- Rapid Elasticity: Computing resources can be promptly and elastically scaled out and in depending on the demand for resources [7]

- Measured Service: Resources can be monitored, controlled, provisioned and charged according to a service level
- $\quad$ agreement which will ensure transparency for the cloud clients and the service providers [7].

\subsection{Cloud Service Models}

Cloud Computing has three service models or three architectural layers as identified by NIST as in Table 1.

Table 1. Cloud Services model

\begin{tabular}{|c|c|}
\hline Services & Description \\
\hline $\begin{array}{l}\text { Software as a } \\
\text { service }(\mathrm{SaaS})\end{array}$ & $\begin{array}{l}\text { This is the highest layer and } \\
\text { comprises a complete application } \\
\text { layer offered as a service, on } \\
\text { demand, via multi-tenancy. For } \\
\text { example, Salesforce, Facebook, } \\
\text { LinkedIn, Intuit, Google Apps and } \\
\text { Microsoft Office Live offer basic } \\
\text { business services such as e-mail } \\
\text { and messaging using the SAAS } \\
\text { model [5]. }\end{array}$ \\
\hline $\begin{array}{l}\text { Platform as a } \\
\text { Service (PaaS) }\end{array}$ & $\begin{array}{l}\text { Consumers using PaaS can } \\
\text { develop and/or deploy applications } \\
\text { by using provider's services and } \\
\text { tools. PaaS providers provide } \\
\text { tools for every phase of software } \\
\text { development and testing which can } \\
\text { be utilised to deploy any service } \\
\text { quickly. Examples include Google } \\
\text { App Engine and Microsoft Azure } \\
\text { [6]. }\end{array}$ \\
\hline $\begin{array}{l}\text { Infrastructure as } \\
\text { a Service (IaaS) }\end{array}$ & $\begin{array}{l}\text { Offers a means of delivering basic } \\
\text { storage and compute capabilities } \\
\text { as standardized services over the } \\
\text { network. Amazon (AWS) and } \\
\text { Rackspace are IaaS providers, } \\
\text { which provide servers, storage and } \\
\text { other computing resources [7]. }\end{array}$ \\
\hline
\end{tabular}

\subsection{Cloud Deployment Models}

There are four deployment models for cloud services; they are with derivative variations that address specific requirements. The four models are listed below:

- Public Cloud: In this model, a single organisation generally owns the infrastructure. The infrastructure is made available for public or other organisations and is leveraged to provide different services. This is currently the most widely used model globally.

- Private Cloud: In this model, the infrastructure is utilised by a single organisation and hence, it is not made available to anyone outside the organisation. The infrastructure can either be managed by the organisation or another 
organisation may manage it on behalf of the first organisation.

- Community Cloud: In the model, the infrastructure is shared among multiple organisations, which may share a set of common goals and requirements among themselves. The infrastructure of this type is managed by different members of the community using a predetermined level of agreement.

- Hybrid Cloud: In this model, the cloud infrastructure is a combination of two or more other cloud models where particular application scenarios prohibit the usage of a certain cloud model.

\section{Cloud in Higher Education Institutions}

Higher Education institutions play an important role in the growth of societies. As with organizations nowadays, universities have become more reliant on Information and Communication Technology (ICT). ICT and internet-based services have to provide their stakeholders with educational services. Cloud computing is likely to be an attractive proposition to start up and small to medium educational establishments. The potential of cloud computing may include but is not limited to increasing service efficiency and cost-savings. An example from the University of California (UC) at Berkeley, found cloud computing to be attractive for use in one of their courses which was focused exclusively on developing and deploying SaaS applications [8].

The Medical College of Wisconsin Biotechnology and Bioengineering Centre in Milwaukee found the use of cloud computing in their research has provided an astounding computing power. Researchers at the centre have been doing protein research which has been made more accessible to scientists from anywhere in the world. This is due largely to renting Google's cloud-based servers [9].

Some universities have adopted cloud computing for economic reasons. The Washington State University's School of Electrical Engineering and Computer Science (EECS) has suffered cuts in its budget. However, the EECS claims that despite the challenging economic climate, cloud computing has actually enabled it to expand the services it offers to faculties and students [9].

Some Universities are facing difficulties to provide scalable and flexible IT services. For instance, in traditional computer labs, there are many challenges present such as, limitation of lab hours and seats during the peak hours, repairing and maintaining computer labs, traveling to and from university, cost of outfitting traditional computer lab (hardware and software). Normally, IT services required by students, researchers and academic are requested from the IT Department, whose job is illustrated in Figure 1.

The IT department provides students, staff, academics and developers with different software and hardware tools. However, in cloud computing all these arrangements can be migrated to the cloud [9]. Figure 2 illustrates an example of how cloud computing is used in the university.

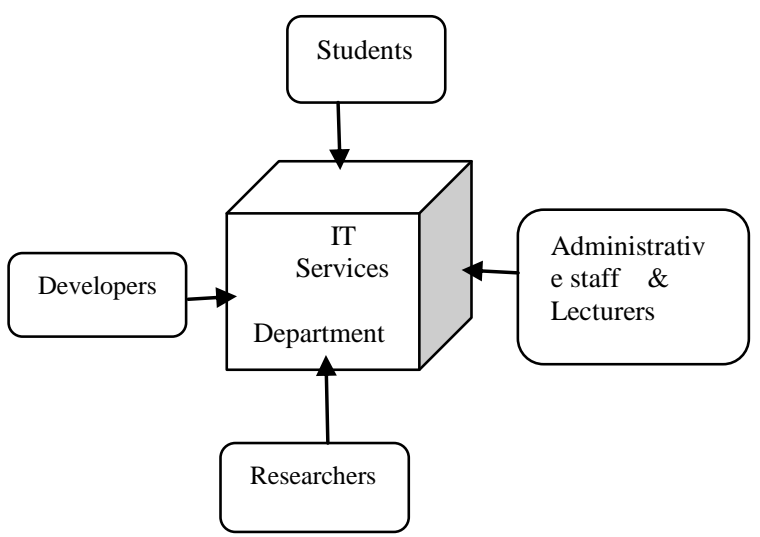

Figure 1. Users of Traditional IT services in a University

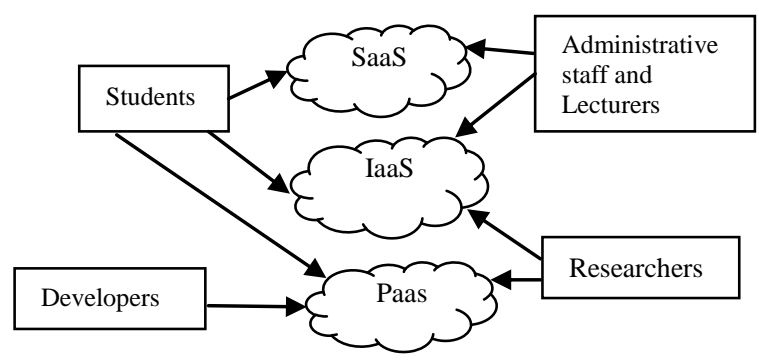

Figure 2. Cloud computing service models in a University

Cloud Computing offers services that enable the universities to concentrate more on teaching and research activities rather than building on complex IT configurations and software systems [10]. It can also be deployed more quickly. Complexity can be reduced with Cloud Computing. Students can exploit different learning tools. Students already use some, such as Google Docs and Office365 and Windows Azure Platform for computer science students. Students can access the learning resources they need from anywhere and at any time with any Internet capable device. Lecturers may experience flexible benefits as the cloud provides an easier platform to prepare their teaching portfolio presentations, lessons, conferences, articles, etc. Researchers may also benefit from the advantages of using the latest technologies and hardware to do their experiments, while paying for using these services only on demand.

Developers can design, build and test applications on the infrastructure of the cloud service provider and produce those applications from cloud provider data centres to the end user [9] System administrators can leverage processing, storage, 
database management and other resources available on the cloud.

\section{Cloud Services in Higher Education}

The trend of educational cloud computing has been adopted by many leading IT companies. Microsoft, Google, Amazon and IBM have provided much initiative to support education institutions with the necessary learning tools. Some of these initiatives are free with no cost. Table 2 shows some of the existing educational clouds and tools. With the availability of content online, it is unnecessarily for lecturers to print teaching materials. Nowadays, students have the choice to access homework assignments, lesson notes, and other materials online with the cloud. Some of the leading cloud services in higher education are described below.

Table 2. Educational cloud-based applications

\begin{tabular}{|c|c|c|}
\hline $\begin{array}{l}\text { Commercial } \\
\text { Product Name }\end{array}$ & $\begin{array}{l}\text { Education cloud } \\
\text { apps }\end{array}$ & Features \\
\hline $\begin{array}{l}\text { Microsoft } \\
\text { Education } \\
\text { Cloud }\end{array}$ & $\begin{array}{l}\text { Microsoft } \\
\text { Live@edu }\end{array}$ & $\begin{array}{l}\text { Website Creation } \\
\text { File sharing } \\
\text { Word processing } \\
\text { Desktop sharing } \\
\text { Resource scheduling }\end{array}$ \\
\hline $\begin{array}{l}\text { Google } \\
\text { Education } \\
\text { Cloud }\end{array}$ & $\begin{array}{l}\text { Google Apps } \\
\text { Education (GAE) }\end{array}$ & $\begin{array}{l}\text { Google Mail } \\
\text { Google Sites } \\
\text { Google Docs } \\
\text { Google Video } \\
\text { Google Calendar } \\
\text { Google Talk }\end{array}$ \\
\hline Earth Browser & Earth Browser & $\begin{array}{l}\text { Provide real } \\
\text { Time data for weather, } \\
\text { geological and other } \\
\text { data }\end{array}$ \\
\hline Socratica & Socratica & $\begin{array}{l}\text { Classrooms in science } \\
\text { to access } \\
\text { Create and study } \\
\text { modules }\end{array}$ \\
\hline VMWare & Virtual Desktop & $\begin{array}{l}\text { Provide Virtual } \\
\text { computers }\end{array}$ \\
\hline $\begin{array}{l}\text { IBM Cloud } \\
\text { Academy }\end{array}$ & $\begin{array}{l}\text { Virtual computing } \\
\text { lab }\end{array}$ & Smart analytics system \\
\hline
\end{tabular}

- Microsoft Education Cloud

Microsoft Education Cloud has been actively developing educational cloud services such as Microsoft Office 365. It provides schools with free email, website with editing and storage facility, instant messaging, web conferencing, and $25 \mathrm{~GB}$ of personal storage. Furthermore, students and faculty are able to use any browser to create documents using Microsoft Office.
The downside to Microsoft 365 is the cost. While a free option is available (with a signed contract), a per-user monthly payment is required to access features such as Office Mobile, Office applications for PC or Mac, unlimited email storage and voicemail. More alarming is Microsoft's inability to ensure $99.9 \%$ uptime without monthly payment.

\section{- Google Education Cloud}

Google Apps for Education is one of the most used application as it does not involve actual cost. It is free with no hidden costs. Some of the feature include cloud email, 30GB of storage, hosting, word processing and collaboration tools. Google is Microsoft's strongest competitor. If it is compared to Microsoft's Office Suite, there is an existing familiarity with many of Google's products such as Gmail, Chat, and Calendar. Nevertheless, the main drawback is that it requires users to have (or create) a Google account. It is compulsory for user of age 13 years old and below to get parent consent.

\section{- Earth Browser}

Earth Browser is a virtual globe software developed by Lunar software. It is available online as a flash application or be installed locally as an application. It focuses mainly on visualising geophysical information such as weather, earthquakes, etc. It shows the earth as satellite images. Earth Browser can be used in real-time. It shows the object in three dimensional model with continuously updates information. The representation of the earth is rendered along with a large data which is said to be accurate. The object can also be rotated and zoomed to a given distance.

\section{- Socratica}

Socratica produces high-quality educational videos for people of all ages. The videos developed are high-definition, clear, concise, and beautiful. Socratica collects and organizes the best free educational videos into topics that can be used by users. Socratica's mission is to organise educational videos. This can be used by users to create optimised learning experience. They have also restricted videos suitable for age groups by having different channels in YouTube.

\section{- Virtual Desktops}

In computing, a virtual desktop is known as another user interface that is able to provide user with the virtual space of a computer's desktop environment through the use of a software application installed in a user's physical computer. Generally, there are two ways to expand the virtual area of the screen. The virtual desktops are switchable allowing user to create virtual copies of their desktop that is switchable. This can be done with open windows existing one desktop. Another approach can expand 
the size of one virtual screen more than the physical viewing device. Usually, navigating an oversized virtual desktop is viewed using scrolling/panning into the subsection of the virtual desktop. One of the most popular VMware product is VMware Horizon 6. It provides a virtual desktop infrastructure (VDI) platform that provides virtualized and remote desktops and applications system through one platform, enabling users access to their online resources through one integrated workspace.

\section{- IBM cloud academy}

IBM cloud academy is a collaborative community of leaders in education. It is intended for educational institutions, with a goal to help reduce costs and optimise services while making information available, and secure if needed. It can also be used to consolidate resources, improve student success, and accelerate scientific discoveries. On the management part, it is expected to add administrative efficiencies, and conserve resources.

These are known as how cloud can help educational institutions to provide services. They are actively integrating cloud technologies into their infrastructures to share best practices in the use of clouds and to collaborate with partners to create innovative cloud technologies and models.

\section{Challenges of Cloud Migration in Saudi Universities}

The following is a list of challenges from literature that can hinder the adoption of cloud computing in higher education in the Saudi universities context. Based on literature reviewed and taking into account the nature of the Saudi higher education context, it can be concluded that the most important challenge for the migration of cloud computing in Saudi Arabia would include the elements summarised below:

- Provision of control, data and service availability issues

Migrating to the cloud primarily focusses on issues of control, loss of data, service and availability. One of the main concerns in higher education is related to who controls the data. Cloud computing makes it possible to deliver everything in digital form. Copyright law and patent law strive to protect the intellectual property of the owners. Course contents, instructional framework and syllabi are made transparent and accessible to all. However, Saudi universities should have cloud providers define their data recovery and business continuity postures in detail, particularly regarding what they are responsible for during a disaster affecting their data centres. Once the location is decided, they need to consider the "availability" part. Authorised users need assured access to information, and cloud storage must be designed to be a robust and continually backed-up environment for data. The cloud has become a data repository but it is also a single point of failure. A loss of internet connectivity anywhere between a university customer and their cloud provider's network will cause interruptions of varying severity. Users of Gmail had to face a service outage in September 2013 for one day due to dual network error and that indicates that even the biggest and most ubiquitous clouds can fail sometimes [11].

\section{- Challenges of applying security protocols during migration}

Privacy and security remain the top concerns for higher educational institutions planning to adopt cloud computing, due to the migration of proprietary and sensitive data outside campus walls. Issues of anonymity, compliance, integrity, reliability and auditability must also be considered in a migration process. Hence, the importance of security within University IT environment is foremost. Nearly onethird of IT professionals in higher education identified potential security breaches as the single biggest barrier to cloud adoption. With so many concerns around security, privacy, and compliance in higher education jurisdiction, it is highly likely that the situation will raise problems with the policy makers and stakeholders in the Saudi context as well [12].

\section{- Legal policy constraints and compliance}

In addition to the usual security concerns for any enterprise, educational institutions, by virtue of their diverse operations, are subject to numerous compliance regimes, and when it comes to compliance, universities can outsource responsibility but cannot outsource accountability [13]. Accountability within Saudi organisations is generally attributed on individual basis which is different from many global enterprise cultures and hence it bears significant importance. Universities, in general, have indirect administrative responsibility for the security of their data and applications and are accountable for data breaches. However, within Saudi context, data privacy is driven strongly by government initiatives directed at the organisation responsible. University's data or applications are stored at remote destinations and Education CIOs need to know where their data will be hosted because any large scale implementation of cloud services by educational establishments may have to wait until law-makers begin to address the legal issues related to privacy and data protection in the context of cloud computing [13]. Again, as the onus of responsibility in Saudi context is more on organisations, the burden of compliance stays with the organisation as well and 
hence there are likely to be accountability issues during the migration process.

Moreover, due to lack of clear legal policies for higher education in Saudi Universities, cloud users and providers will need to be more careful in their approach to cloud computing in order to prevent disasters and need to make sure that due diligence is carried out. This involves developing an institutionwide cloud strategy to help the institution select the right sourcing and solution strategies. Saudi universities are subject to numerous state and national laws covering data on academic grades, health records and financial aid, among other things. Saudi Arabia has very strict rules about cross-border transfers of personal information, and complying with those rules can be challenging in the virtual world of the cloud [14].

Because data centres powering cloud computing platforms frequently exist in multiple nations, this triggers cross-border issues that can pose additional complex regulatory questions, or outright barriers, for Saudi universities. Institutions holding sensitive government contracts, for example, for those subject to export controls over their research materials and intellectual property cannot permit digital material pertaining to these leave the country.

- Vendor lock-in, loss of service, SLA, latency, and performance

Unlike traditional software packages that can be installed on a local computer and are available as long as the operating system supports them, cloudbased applications are services offered by companies and service providers in real time. Saudi Universities need to be able to trust that the service provider will continue to be there, even in face of changing market. Typical cloud agreements define service level agreements (SLAs) establishing providers' expected uptime and performance measurements. Universities should look at those measurements and understand what they actually mean in terms of enduser experience and the customer's operations [13].

\section{- Cross-platform interoperability}

Higher education institutions serve students, faculty staff and admin staff who come to campus with their own devices and expectations about how and when they want to use them. BYOD: Bring your own device initiative poses many challenges to IT departments. IT staff must now provide greater interoperability between campus and stakeholder platforms; 24/7 access to secure, reliable networks; and the ability to create, deliver, and share content campus-wide on any number of devices. Cloud computing is now as much about meeting student needs as it is about running an efficient campus [15]. Within the Saudi context, staff members and particularly students are likely to have a diverse range of machines. Hence, an email client, for instance, from a Mac OS must not have any compatibility issues with a MS Exchange Server hosted on the cloud platform. Thus, cross-platform compatibility must be considered during the migration process as well.

\section{- Users acceptance and awareness of new cloud-} driven paradigm

User acceptance and awareness involves developing a staffing and organizational model to accommodate the changing IT environment and facilitate openness. This will provide agility to increase the awareness and training sessions to support university stakeholders. Another challenge to widespread adoption of cloud computing is the vagueness and resistance that may exist among staff. It is widely known that introducing a new innovation can result in employee resistance, particularly if there is a lack of understanding of the change or indeed a lack of knowledge on how it will affect their work, e.g. may be fear of eventual downsizing. Similarly, in the Saudi context, staff members are known to assume positions for decades. Hence, they get used to company cultures, technological routines and standards. Therefore, any potential migration to a seemingly new paradigm is likely to cause resistance as senior decision-makers in organisations need to prepare employees for this new learning curve by providing training and communication in advance of cloud implementation [16].

\section{The Proposed Conceptual Framework}

Critical Success Factors (CSFs) are defined by many researchers in the past but it was introduced first by Rockart in 1978 [17]. In this study, they can be defined as 'Those enablers that should be guaranteed by university for successful migration of their traditional ICT educational services to the cloud based services' [18]. The proposed framework is constructed to examine the most important success factors that will enable the universities IT decision makers and Staff to migrate to the cloud. The framework integrates cultural aspects related to the success factors for implementing ICT projects in the Saudi higher education [5] context along with redefined success factors from the characteristics of cloud computing paradigm (Privacy, Security, Interoperability, Internet bandwidth and Service Level Requirements) [16]. The research conceptual framework has two CSFs categories: Technological CSFs and Organizational CSFs as Shown in

Figure 3.

\subsection{Technological CSFs}


This category illustrates the technological aspects that can facilitate the success of the migration process to the cloud [19].

- Reliability: The University cloud-based services should be reliable and continuously available by providing redundant services, but the possibility still exists that the system could crash and leave clients with no way to access their saved data. Many existing cloud infrastructures leverage commodity hardware that is known to fail unexpectedly. A loss of Internet connectivity anywhere between a university customer and their cloud provider's network will cause interruptions of varying severity. It is important for the service to be reliable, if it is available. So without availability, reliability cannot be achieved. A cloud service should first be available in order to be reliable.

- Interoperability: A universal set of standards and interfaces has not yet been defined for cloud based services, which result in a significant risk of vendor lock-in. Higher education institutions should make sure that the Cloud based IT solutions must be interoperable and compatible between different providers; 24/7 access to secure, reliable networks; and the ability to create, deliver, and share content campus-wide on any number of devices.

- Security \& Privacy: Privacy and security are the top concerns for higher educational institutions planning to adopt cloud computing, due to the migration of sensitive data such as students' records, Researchers' Patents and Intellectual properties outside campus walls. Hence the importance of security and information privacy within University IT environment is crucial [20].

- Disaster recovery: Saudi universities should ensure data recovery and business continuity back up plans, particularly regarding what they are responsible for during a disaster affecting their data centers. Universities have indirect administrative responsibility for the security of their data and applications and are accountable for data breaches and disaster recovery.

- Network Bandwidth: Cloud computing is a stateless system, as is the Internet in general. In order for communication to survive on a distributed system, it is necessarily unidirectional in nature. Most of remote requests used in the cloud is through HTTP messages like PUT and GET. The HTTP requests reach the provider and the service provider then sends a response. Low bandwidth would increase the latency of communications and the service would become very slow, if bandwidth is not increased. Therefore, Saudi universities should increase the
Internet bandwidth in order to provide good cloud-base services to their stakeholders.

- Disaster recovery: Saudi universities should ensure data recovery and business continuity back up plans, particularly regarding what they are responsible for during a disaster affecting their data centers. Universities have indirect administrative responsibility for the security of their data and applications and are accountable for data breaches and disaster recovery.

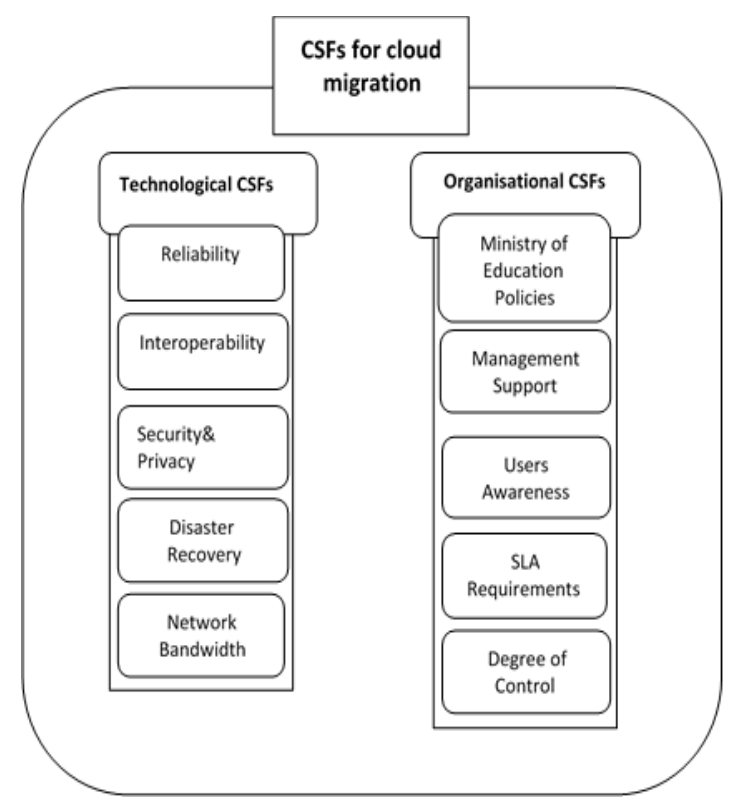

Figure 3. Cloud Migration CSFs Framework

\subsection{Organizational CSFs}

This category describes the aspects that are related to University culture and operational roles, which can contribute to the success of the migration process to the cloud [19].

- Ministry of Education Policies: Since cloud computing is a relatively new IT Paradigm, it will require changes to be made in Ministry of Higher Education policies and regulations and comply with legislations in Saudi government to ensure the safety of stakeholders' information. Saudi universities need to improve data policies in order to protect their sensitive information. This involves developing institution wide cloud policies to help the institutions select the right sourcing and solutions that comply with the regulations in Saudi Arabia.

- Top Management Support: From a strategic perspective, the successful implementation of cloud solutions in Saudi Arabia depends on the capabilities of top leadership or management to drive the change from traditional deployment to 
cloud adoption through an official pro-cloud strategy. The decision makers' awareness and consensus is vital. Their support will ensure what cloud services are needed and what type of cloud deployment is best for higher education settings. In order to do that the decision makers have to understand the benefits of cloud-based services, the value they can add to the educational services and how to migrate to cloud computing environment.

- Users' awareness: Successful utilization of any new technology requires proper plan to educate and increase the awareness of the stakeholders dealing with the technology and how to develop cloud solutions to serve the educational entities. Therefore, Saudi university IT department should provide their IT staff with training session on how to run cloud-based services and then introduce the cloud computing services to their stakeholders (academics and Students) and provide them with the guide needed in order to use the cloud services properly. The motivation of academics to acquire the required competencies and to make use of cloud based IT for innovation and research is greatly influenced by encouragement from higher authorities and decision makers of Saudi universities. Saudi Higher Education institutions should offer incentives for their people to access cloud based services within a well-defined institutional framework. University decision makers and CIOs should make cloud solutions a part of personal work routines and teaching processes [5].

- Customizable SLA requirements: Saudi Universities need to be able to ensure that the service provider will continue to be there, even in face of changing market. Service level agreements (SLAs) are the contracts between the users of cloud services and the provider and it contains the expected uptime and performance of those services. Universities should consider those measurements and prepare list of customized requirements for each services they use and it needs to collect each department requirements in order to indicate them in the SLA to cover the end-user experience and the customer's operations.

- Degree of Control: The amount of control that the user has over the cloud environment varies greatly. In traditional IT environment, the consumer had full control over the services accessed. But the same is not true for the cloud services. For that reason, there is loss of control when universities migrate to the cloud, and it is the duty of the university CIO or management to make sure that the control is given to the right provider or decide what should be under their control and what can be migrated.

\section{Conclusion and Future Work}

Cloud computing is a new ICT paradigm to deliver IT services to users as utility over the Internet. The cloud paradigm is well known for its scalability, which could provide direct and immediate benefits to rapidly expanding higher education organizations' services. Moreover, cloud computing for higher education institutions can provide flexible, on demand resources to multiple users at any time based on the share of services used over a Pay As You Go model. However, despite the aforementioned advantages of cloud services, the migration process to cloud based services within the Saudi context faces many challenges and these challenges vary according to the cultural aspects of the technology, organization, legislation and their requirements and infrastructure. The aim of this paper is to form a conceptual framework in order to investigate the most CSFs that can contribute towards successful migration to the cloud in the Saudi higher education settings.

Therefore, as this is an ongoing research, the future work will be concentrated on reviewing and confirming the proposed CSFs by utilizing the sequential methodological triangulation approach. The cross confirmation of the framework will involves IT interviews and online questionnaire with experts working in the deanship of Information Communication at different Saudi Universities. Moreover, based on the confirmed CSFs, an instrument will be developed using goal question metrics approach, which will be used as measuring tool for estimating the readiness of the Saudi universities that intend to migrate to the educational cloud services. The usefulness and accuracy of the developed instruments will be validated afterwards with three case studies in three startup Saudi universities.

\section{References}

[1] R. Buyya et al., "Cloud computing and emerging IT platforms: Vision, hype, and reality for delivering computing as the 5th utility," Futur. Gener. Comput. Syst., vol. 25 , no. 6, p. 17, 2009

[2] M. Alabbadi, "Cloud computing for education and learning: Education and learning as a service (ELaaS)," 14th Int. Conf. Interact. Collab. Learn., no. September, pp. 589-594, 2011.

[3] A. Q. Naif Jabil, "The Benefits and Barriers of Elearning in Higher Education in Saudi Arabia," 2013. .

[4] A. N. Tashkandi and I. M. Al-Jabri, "Cloud computing adoption by higher education institutions in Saudi Arabia: an exploratory study," Cluster Comput., vol. 18, no. 4, pp. 1527-1537, 2015.

[5] M. S. A. A. S. A.-M. Abeer I. ALdayel, "The Critical Success Factors of ERP implementation in Higher 
Education in Saudi Arabia: A Case StudyNo Title," J. Inf. Technol. Econ. Dev., vol. 2, no. 2, pp. 1-16, 2011.

[6] R. Buyya, C. S. Yeo, and S. Venugopal, "MarketOriented Cloud Computing: Vision, Hype, and Reality for Delivering IT Services as Computing Utilities," 2008 10th IEEE Int. Conf. High Perform. Comput. Commun., pp. 513, Sep. 2008.

[7] P. Mell and T. Grance, “The NIST Definition of Cloud Computing Recommendations of the National Institute of Standards and Technology," Nist Spec. Publ., vol. 145, p. 7, 2011.

[8] A. Alshwaier, "A New Trend for E-Learning in KSA Using Educational Clouds," Adv. Comput. An Int. J., vol. 3, no. 1, pp. 81-97, 2012.

[9] N. Sultan, "Cloud computing for education: A new dawn?,” Int. J. Inf. Manage., vol. 30, pp. 109-116, 2010.

[10] Y. Y. Al Alhareth et al., "Review of Women's Higher Education in Saudi Arabia," 2015.

[11] A. Shakeabubakor, "Cloud Computing Services and Applications to Improve Productivity of University Researchers," Int. J. Inf. Electron. Eng., vol. 5, no. 2, pp. 153-157, 2015.

[12] A. Albugmi, M. O. Alassafi, R. J. Walters, and G. B. Wills, "Data Security in Cloud Computing," In, Fifth Int. Conf. Futur. Gener. Commun. Technol. (FGCT 2016), Luton, GB, 17 - 19 Aug 2016., pp. 55-59, 2016.

[13] Cloud Security Alliance, "Security Guidance for Critical Areas of Focus in Cloud Computing V3.0," 2011.

[14] N. Koch, "The shifting geopolitics of higher education: Inter/nationalizing elite universities in Kazakhstan, Saudi Arabia, and beyond," Geoforum, vol. 56, pp. 46-54, 2014.

[15] T. Dillon, C. W. C. Wu, and E. Chang, "Cloud Computing: Issues and Challenges," Adv. Inf. Netw. Appl. (AINA), 2010 24th IEEE Int. Conf., pp. 27-33, 2010.

[16] A. Alharthi, F. Yahya, R. J. Walters, and G. B. Wills, "An Overview of Cloud Services Adoption Challenges in Higher Education Institutions," in Proceedings of the 2nd International Workshop on Emerging Software as a Service and Analytics, 2015, pp. 102-109.

[17] G. Carroll and J. Delacroix, "The changeing role of the Inforamtion System executives: A critical success factors perspective," 1982.

[18] A. Alharthi, M. O. Alassafi, R. J. Walters, and G. B. Wills, "An exploratory study for investigating the critical success factors for cloud migration in the Saudi Arabian higher education context," Telemat. Informatics, vol. 34, no. 2,2017

[19] A. Alharthi, M. O. Alassafi, R. J. Walters, and G. B. Wills, "Towards a framework to enable the migration process to eduactional clouds in Saudi higher education," i-Society Conferance IEEE Adv. Technol. Humanit., no. 1, pp. $1-4,2016$.

[20] Madini O. Alassafi, A. Alharthi, R. J. Walters, and G. B. Wills, "Security Risk factors that influence Cloud Computing Adoption in Saudi Arabia Government Agencies," i-Society Conferance IEEE Adv. Technol. Humanit., vol. 1, pp. 1-4, 2016

\section{Acknowledgment}

We acknowledge the award of University of Jeddah scholarship to Abdulrahman Alharthi and the Saudi Arabian Cultural bureau in London (SCAB) for allowing the research to be funded and undertaken. 